\title{
Propriétés chimiques de la poudre de lait écrémé fabriquée selon la méthode de séchage à double effet
}

\author{
par \\ J. DE VILDER*
}

\section{INTRODUCTION}

Une étude précédente [5] autorisait la conclusion que le séchage à double effet, en conditions de travail normales, influe favorablement sur les propriétés physiques et sur la structure de la poudre de lait entier. L'auteur constatait toutefois qu'une température de séchage en « lit fluidisé » supérieure à $95^{\circ} \mathrm{C}$, appliquée pendant $4 \mathrm{~min}$, annihilait partiellement cette influence positive.

Le commerce des poudres de lait accorde autant d'importance à certaines caractéristiques chimiques qu'aux propriétés physiques de ces produits. Les principales sont à la base de la classification des poudres en fonction des traitements thermiques appliqués : les propriétés réductrices, l'intensité de la réaction de Maillard, le nombre de groupes SH libres, les teneurs totales en groupes thiol et en liaisons disulfure (groupes SS). Complémentairement, on attache aussi de l'importance à la stabilité à la chaleur. Une étude comparative de poudres séchées respectivement par la méthode classique et par la méthode à double effet a été entreprise afin de procurer plus d'informations sur ces caractéristiques. Le séchage à double effet était réalisé avec différentes intensités de séchage en "lit fluidisé ".

\section{DONNEES DE LA LITTERATURE}

La classification des poudres en fonction des traitements thermiques appliqués fournit une indication précise sur la dénaturation

\footnotetext{
* Station Laitière de l'Etat, Melle, Centre de Recherches Agronomiques de l'Etat, Gand (Belgique).
} 
des protéines lactosériques au cours des divers échauffements [6] et l'indice de dénaturation est un paramètre très important pour l'évaluation de quelques caractéristiques fonctionnelles des poudres de lait.

Les propriétés réductrices d'une poudre de lait sont en rapport étroit avec sa teneur en antioxydants. Kumetat et al. [11] ont signalé dès 1956 que les poudres de lait écrémé à dissolution instantanée avaient un pouvoir réducteur nettement plus élevé que celui des poudres de lait normales. Ces auteurs avaient toutefois remarqué que les propriétés réductrices étaient influencées diversement, selon le procédé mis en œuvre. Selon Crowe et al. [3] et Henry et al. [8], cette caractéristique serait également en étroit rapport positif avec l'intensité de la réaction entre acides aminés et hydrates de carbone (réaction de Maillard). Keeney et Bassette [10] et Keeney [9] ont observé que certains procédés commerciaux d' « instantanéisation » déclenchent un brunissement nuisible à la qualité organoleptique des poudres, observation qui n'a toutefois pas été confirmée par Mann et Mc Intire [13]. Keeney et Bassette [10] attribuent l'intensification de la réaction de Maillard au processus mis en œuvre pendant l'agglomération. La poudre réhumidifiée subit un séchage final de quelques minutes à l'air chaud, ce qui peut entraîner une nette accentuation du brunissement.

Au cours du séchage à double effet, des poudres à relativement haute teneur en eau ( 6 à 8 p. 100) sont également soumises à un séchage final dans un " lit fluidisé ». Importante à ce propos est une observation faite par Eggum et al. [7], selon laquelle la réaction de Maillard se déroule le mieux à des teneurs en humidité situées entre 7 et 15 p. 100. De hautes températures étant appliquées pour le séchage en "lit fluidisé ", le risque de brunissement n'est nullement imaginaire.

Les groupes SH libres sont considérés comme des antioxydants naturels. Dans le lait non chauffé, ils sont masqués en raison de la structure des protéines [12]. Ils peuvent toutefois être libérés par l'échauffement et devenir ainsi disponibles sous une forme très réactive. Sous l'effet de la chaleur, les groupes $\mathrm{SH}$ forment très facilement des liaisons disulfure (groupes SS). L'exemple le mieux connu de cette réaction est celle de la $\beta$-lactoglobuline avec la caséine $x$. Selon les propriétés fonctionnelles requises des poudres, ces réactions sont tantôt souhaitées, tantôt indésirables.

\section{MATERIEL ET METHODES}

\section{III.1. Fabrication des poudres de lait écrémé}

Tous les essais ont été effectués dans l'installation semi-industrielle de la Station Laitière de l'Etat. 
Le lait écrémé était concentré dans une installation à double effet. Selon l'essai, il avait subi un préchauffage à $75^{\circ} \mathrm{C}, 30 \mathrm{~s}$ ou à $90^{\circ} \mathrm{C}, 30 \mathrm{~s}$. Dans les procédés de séchage classique, ces préchauffages donnent des poudres de lait appartenant respectivement aux classes "low heat " et " medium heat ".

La dessiccation proprement dite a été réalisée dans une installation semi-industrielle d'atomisation par centrifugation, capable d'évaporer 35 à 401 d'eau par heure. La vitesse tangentielle de la roue d'atomisation était de $135 \mathrm{~m} / \mathrm{s}$ dans tous les essais. La température de l'air entrant était toujours de 195 à $200^{\circ} \mathrm{C}$ et la température de l'air sortant de 74 à $76^{\circ} \mathrm{C}$. On obtenait ainsi des poudres à teneur en eau de 6,8 à 7,4 p. 100 .

Le séchage final s'effectuait dans une installation de séchage à " lit fluidisé » couplée à la tour de séchage. En modifiant le volume de la chambre de séchage et la température de l'air sécheur on appliquait pendant 4 ou $8 \mathrm{~min}$ des intensités de séchage de 74 à $98^{\circ} \mathrm{C}$.

Chaque essai a consisté à fabriquer une poudre de lait écrémé dans les conditions classiques, en partant d'un même concentré, mais à des températures d'air entrant et sortant de respectivement $195^{\circ} \mathrm{C}$ et $90^{\circ} \mathrm{C}$.

Les résultats mentionnés dans les tableaux représentent chaque fois la moyenne de trois séries d'essais distinctes, exécutées dans des conditions à peu près identiques.

\section{III.2. Méthodes de détermination}

La dénaturation des protéines du sérum a été déterminée selon la méthode WPNI (Whey Protein Nitrogen Index) et celle de l'indice de caséine. La valeur du WPNI [1] donne la teneur en protéines sériques non dénaturées de $1 \mathrm{~g}$ de poudre; elle permet de distinguer trois classes de poudres :

- les poudres " low heat» : WPNI d'au moins $6,0 \mathrm{mg} \mathrm{N} / \mathrm{g}$;

- les poudres "medium heat »: WPNI de 1,5 à $6,0 \mathrm{mg} \mathrm{N} / \mathrm{g}$;

- les poudres "high heat" : WPNI de moins de 1,5 mg N/g.

La détermination de l'indice de caséine consiste à précipiter la caséine et les protéines sériques dénaturées d'une solution à 10 p. 100 de poudre de lait, à $\mathrm{pH}$ 4,6. Le résultat, exprimé en p. 100 de l'azote total, sert également à définir la classe de la poudre en fonction des traitements thermiques appliqués. Cette classification est contrôlée ensuite, à titre complémentaire, par l'électrophorèse sur gel de polyacrylamide selon la méthode de Assenat [2].

On détermine ainsi dans quelle mesure les protéines sériques ont résisté à l'ensemble des traitements thermiques.

Les propriétés réductrices et l'intensité de la réaction de Maillard ont été déterminées respectivement par les méthodes décrites par Robbertsen et Radema [15] et par Samuelsson et Nielsen [16]. Les 
premières sont exprimées en quantités équivalentes de chlorhydrate de cystéine par gramme de poudre. L'intensité de la réaction de Maillard est révélée par la teneur en hydroxyméthylfurfural (HMF), produit intermédiaire de l'interaction des sucres et des protéines. Le résultat est exprimé en concentrations molaires de HMF dans une solution à 9 p. 100 de poudre.

Les groupes $\mathrm{SH}$ libres ont été détectés par la méthode de Lyster [12]. Leur concentration est exprimée en $\mathrm{mM}$ dans une solution à 9 p. 100 de poudre. Les teneurs en groupes SH et en groupes SS ont été déterminées par la méthode de Mrowetz et al. [14], dont les résultats sont exprimés en $\mathrm{mg} \mathrm{SH}$ ou SS par gramme de protéines.

La stabilité à la chaleur a été déterminée par la méthode de Davies et White [4] à $135^{\circ} \mathrm{C}$ pour le lait reconstitué $(9 \mathrm{p} .100$ d'extrait sec) à $120^{\circ} \mathrm{C}$ pour le lait recombiné (18 p.100 d'extrait $\mathrm{sec}$ ).

\section{RESULTATS ET COMMENTAIRE}

\section{IV.1. L'indice d'azote des protéines sériques (WPNI) et l'indice de caséine}

On sait que dans la fabrication classique des poudres de lait, la dénaturation des protéines sériques est déterminée principalement par le préchauffage du lait et, dans une faible mesure, par les autres traitements thermiques [6]. La bibliographie ne fait aucune allusion aux influences que le séchage à double effet pourrait exercer sous ce rapport.

Le tableau 1 reproduit les résultats moyens de trois séries d'essais, au cours desquelles du lait écrémé a été concentré à température moyenne (conditions medium heat). Différentes températures d'échauffement en "lit fluidisé ", allant de 74 à $95^{\circ} \mathrm{C}$, ont été appliquées pendant $4 \mathrm{~min}$ au cours du séchage par atomisation selon le système à double effet. Les poudres prélevées en aval du " lit fluidisé " avaient des teneurs moyennes en eau respectivement de 4,17, 3,88 et 2,97 p. 100 selon qu'elles avaient été séchées, pendant $4 \mathrm{~min}$, à des températures d'air de $74-77^{\circ} \mathrm{C}, 81-83^{\circ} \mathrm{C}$ ou $91-95^{\circ} \mathrm{C}$. La poudre obtenue par séchage à simple effet contenait 4,07 p. 100 d'eau.

Ces résultats montrent que les différents traitements thermiques en lit fluidisé n'ont eu aucune influence sur le WPNI et sur l'indice de caséine. Il n'y a pas, non plus, de nette distinction entre la poudre de référence, fabriquée dans les conditions classiques, et les poudres fabriquées selon le procédé à double effet. Comme ces poudres avaient été concentrées en conditions « medium heat », les protéines sériques les plus sensibles à la chaleur (immunoglobulines et $\beta$-lactoglobuline) avaient déjà, de ce fait, subi une certaine dénaturation. Ces essais ont donc été répétés avec une concentration en conditions 


\section{TABLEAU 1}

Dénaturation des protéines du sérum en cas de chauffage à simple ou à double effet.

Préchauffage de la concentration : $90^{\circ} \mathrm{C} / 30 \mathrm{~s}$

\begin{tabular}{|c|c|c|c|}
\hline $\begin{array}{l}\text { Température de l'air } \\
\text { sécheur en } \\
\text { "lit fluidisé " }\end{array}$ & Echantillonnage & $\begin{array}{l}\text { WPNI } \\
\text { mg N/g } \\
\text { poudre }\end{array}$ & $\begin{array}{l}\text { Indice } \\
\text { de caséine } \\
\text { p. } 100\end{array}$ \\
\hline 74 à $77^{\circ} \mathrm{C} / 4 \mathrm{~min}$ & $\begin{array}{l}\text { avant " lit fluidisé » } \\
\text { après « lit fluidisé » }\end{array}$ & $\begin{array}{l}4,7 \\
4,5\end{array}$ & $\begin{array}{l}86,7 \\
86,6\end{array}$ \\
\hline 81 à $83^{\circ} \mathrm{C} / 4 \min$ & $\begin{array}{l}\text { avant « lit fluidisé » } \\
\text { après « lit fluidisé » }\end{array}$ & $\begin{array}{l}4,3 \\
4,5\end{array}$ & $\begin{array}{l}86,8 \\
86,6\end{array}$ \\
\hline 91 à $95^{\circ} \mathrm{C} / 4 \mathrm{~min}$ & $\begin{array}{l}\text { avant " lit fluidisé » } \\
\text { après " lit fluidisé " }\end{array}$ & $\begin{array}{l}4,5 \\
4,6\end{array}$ & $\begin{array}{l}86,6 \\
86,7\end{array}$ \\
\hline $\begin{array}{c}\text { Poudre séchée en simple } \\
\text { effet }\end{array}$ & en aval de la tour & 4,4 & 86,8 \\
\hline
\end{tabular}

TABLEAU 2

Dénaturation des protéines du sérum en cas de chauffage à simple ou à double effet. Préchauffage de la concentration : $75^{\circ} \mathrm{C} / 30 \mathrm{~s}$

\begin{tabular}{|c|c|c|c|}
\hline $\begin{array}{c}\text { Température de l'air } \\
\text { sécheur en } \\
\text { « lit fluidisé " }\end{array}$ & Echantillonnage & $\begin{array}{l}\text { WPNI } \\
\mathrm{mg} \mathrm{N} / \mathrm{g} \\
\text { poudre }\end{array}$ & $\begin{array}{l}\text { Indice de } \\
\text { caséine } \\
\text { p. } 100\end{array}$ \\
\hline 74 à $78^{\circ} \mathrm{C} / 4 \mathrm{~min}$ & $\begin{array}{l}\text { avant « lit fluidisé » } \\
\text { après « lit fluidisé » }\end{array}$ & $\begin{array}{l}7,1 \\
7,2\end{array}$ & $\begin{array}{l}82,2 \\
83,4\end{array}$ \\
\hline 85 à $90^{\circ} \mathrm{C} / 8$ mïn & $\begin{array}{l}\text { avant « lit fluidisé » } \\
\text { après « lit fluidisé » }\end{array}$ & $\begin{array}{l}7,2 \\
7,1\end{array}$ & $\begin{array}{l}82,2 \\
82,2\end{array}$ \\
\hline 96 à $98^{\circ} \mathrm{C} / 4 \mathrm{~min}$ & $\begin{array}{l}\text { avant "lit fluidisé " } \\
\text { après " lit fluidisé " }\end{array}$ & $\begin{array}{l}7,4 \\
7,3\end{array}$ & $\begin{array}{l}81,2 \\
82,0\end{array}$ \\
\hline $\begin{array}{c}\text { Poudre séchée en simple } \\
\text { effet }\end{array}$ & en aval de la tour & 7,4 & 81,8 \\
\hline
\end{tabular}




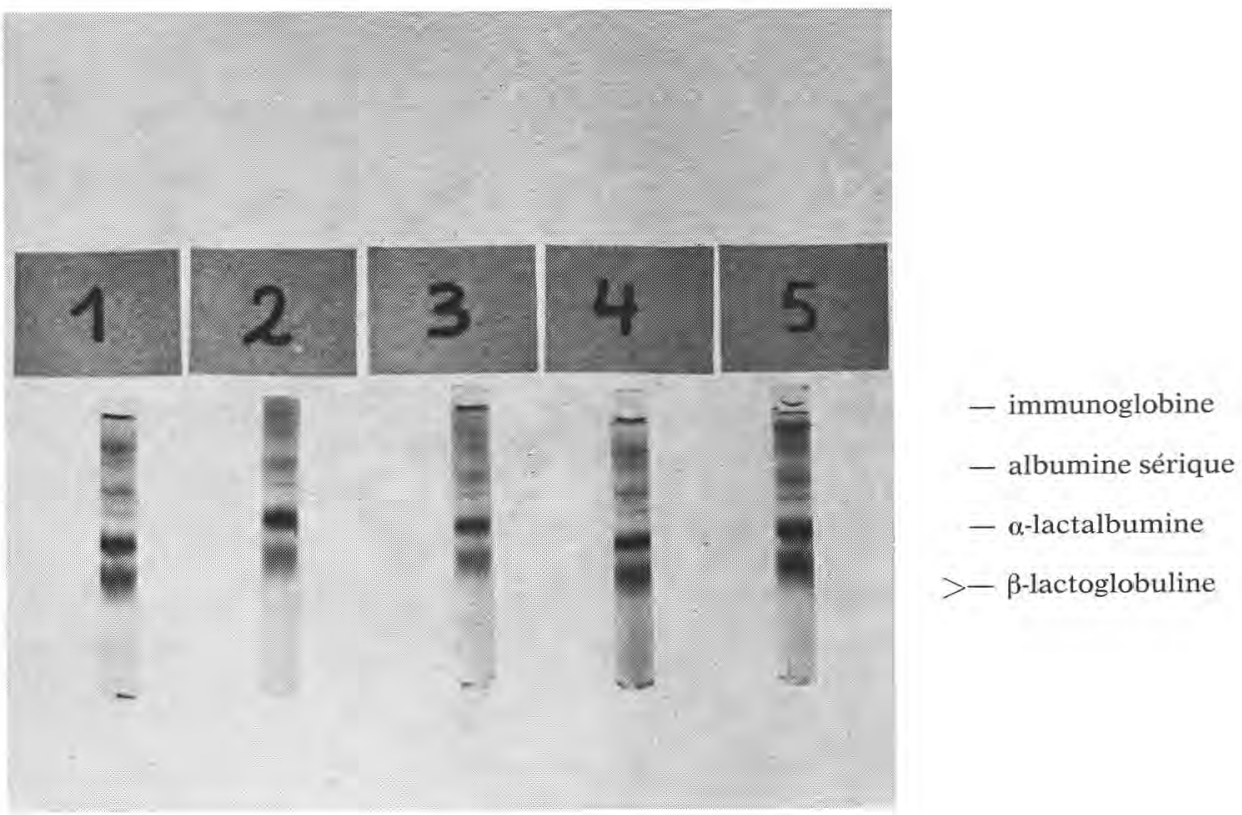

fig. 1

Electrophorèse sur gel de polyacrylamide des protéines du sérum

(poudres du tableau 2)

1. Poudre séchée en simple effet.

2. Echantillon prélevé en amont du "lit fluidisé ".

3. Poudre séchée en double effet: température de l'air sécheur en "lit fluidisé » : 74 à $78^{\circ} \mathrm{C} / 4 \mathrm{~min}$.

4. Poudre séchée en double effet : température de l'air sécheur en " lit fluidisé " : 85 à $90^{\circ} \mathrm{C} / 8 \mathrm{~min}$.

5. Poudre séchée en double effet : température de l'air sécheur en "lit fluidisé »: 96 à $98^{\circ} \mathrm{C} / 4 \mathrm{~min}$.

"low heat » et, de plus, des échauffements en « lit fluidisé » encore plus intenses que ceux décrits ci-dessus (tab. 2). Les poudres prélevées en aval du " lit fluidisé " contenaient respectivement 4,21, 2,14 et $2,60 \mathrm{p} .100$ d'eau selon qu'elles avaient subi un séchage final de $4 \min$ à $74-78^{\circ} \mathrm{C}$, de $8 \min$ à $85-90^{\circ} \mathrm{C}$ ou de $4 \min$ à $96-98^{\circ} \mathrm{C}$. La poudre obtenue par séchage à simple effet contenait 3,88 p. 100 d'eau.

Les résultats montrent également que le séchage à double effet, considéré dans son ensemble, et le séchage en "lit fluidisé ", considéré séparément, n'exercent aucune influence. Les différences minimes, qui se manifestent parfois, restent dans les limites de reproductibilité des méthodes appliquées. Les chiffres reproduits ici sont également les moyennes de trois essais séparés. 
La séparation par électrophorèse des protéines du sérum a permis de confirmer les résultats précédents. La figure 1 montre que même un échauffement en " lit fluidisé », par air sécheur à 96-98 C et d'une durée de $4 \mathrm{~min}$, n'a pas eu d'influence évidente sur la fraction la plus sensible à la chaleur, c'est-à-dire les immunoglobulines.

\section{IV.2. Propriétés réductrices et réaction de Maillard}

Le tableau 3 donne les résultats relatifs aux propriétés réductrices, ainsi qu'à l'intensité de la réaction de Maillard (teneur en HMF) des poudres concentrées en conditions "medium heat ". Ces chiffres sont les moyennes de trois séries d'essais séparées.

\section{TABLEAU 3}

Influence du séchage à simple ou à double effet sur les propriétés réductrices et sur la teneur en hydroxyméthylfurfural.

Préchauffage de la concentration : $90^{\circ} \mathrm{C} / 30 \mathrm{~s}$

\begin{tabular}{|c|c|c|c|}
\hline $\begin{array}{l}\text { Température } \\
\text { de l'air sécheur en } \\
\text { " lit fluidisé " }\end{array}$ & Echantillonnage & $\begin{array}{c}\text { Propriétés } \\
\text { réductrices } \\
\text { chlorhydrate de } \\
\text { cystéine } \\
10-4 \mathrm{~mol} / \mathrm{g} \text { poudre }\end{array}$ & $\begin{array}{c}\text { Réaction } \\
\text { de Maillard } \\
\text { hydroxyméthyl- } \\
\text { furfural } 10-5 \mathrm{M}\end{array}$ \\
\hline 74 à $77^{\circ} \mathrm{C} / 4 \mathrm{~min}$ & $\begin{array}{l}\text { avant " lit fluidisé " } \\
\text { après " lit fluidisé " }\end{array}$ & $\begin{array}{l}0,184 \\
0,183\end{array}$ & $\begin{array}{l}0,293 \\
0,325\end{array}$ \\
\hline 81 à $83^{\circ} \mathrm{C} / 4 \min$ & $\begin{array}{l}\text { avant " lit fluidisé " } \\
\text { après " lit fluidisé " }\end{array}$ & $\begin{array}{l}0,188 \\
0,174\end{array}$ & $\begin{array}{l}0,308 \\
0,327\end{array}$ \\
\hline 91 à $95^{\circ} \mathrm{C} / 4 \mathrm{~min}$ & $\begin{array}{l}\text { avant " lit fluidisé " } \\
\text { après " lit fluidisé » }\end{array}$ & $\begin{array}{l}0,184 \\
0,170\end{array}$ & $\begin{array}{l}0,298 \\
0,316\end{array}$ \\
\hline $\begin{array}{l}\text { Poudre séchée en } \\
\text { simple effet }\end{array}$ & en aval de la tour & 0,171 & 0,297 \\
\hline
\end{tabular}

Ils montrent que les différentes intensités de séchage en « lit fluidisé » n'ont pas influé sur les caractéristiques susdites. En outre, on ne perçoit aucune différence entre la poudre obtenue par séchage à simple effet et les autres échantillons.

Le tableau 4 reproduit les résultats moyens d'essais analogues, où l'on était parti de concentrés préparés en condition "low heat ". Il établit une fois de plus que, comparés au procédé de séchage clas- 


\section{TABLEAU 4}

Influence du séchage à simple ou à double effet sur les propriétés réductrices et sur la teneur en hydroxyméthylfurfural.

Préchauffage de la concentration : $75^{\circ} \mathrm{C} / 30 \mathrm{~s}$

\begin{tabular}{|c|c|c|c|}
\hline $\begin{array}{l}\text { Température } \\
\text { de l'air sécheur en } \\
\text { "lit fluidisé " }\end{array}$ & Echantillonnage & $\begin{array}{c}\text { Propriétés } \\
\text { réductrices } \\
\text { chlorhydrate de } \\
\text { cystéine } \\
10-4 \mathrm{~mol} / \mathrm{g} \text { poudre }\end{array}$ & $\begin{array}{c}\text { Réaction } \\
\text { de Maillard } \\
\text { hydroxyméthyl- } \\
\text { furfural } 10-5 \mathrm{M}\end{array}$ \\
\hline 74 à $78^{\circ} \mathrm{C} / 4 \mathrm{~min}$ & $\begin{array}{l}\text { avant " lit fluidisé " } \\
\text { après " lit fluidisé " }\end{array}$ & $\begin{array}{l}0,160 \\
0,159\end{array}$ & $\begin{array}{l}0,228 \\
0,243\end{array}$ \\
\hline 85 à $90^{\circ} \mathrm{C} / 8 \mathrm{~min}$ & $\begin{array}{l}\text { avant "lit fluidisé » } \\
\text { après " lit fluidisé » }\end{array}$ & $\begin{array}{l}0,186 \\
0,180\end{array}$ & $\begin{array}{l}0,203 \\
0,198\end{array}$ \\
\hline 96 à $98^{\circ} \mathrm{C} / 4 \mathrm{~min}$ & $\begin{array}{l}\text { avant "lit fluidisé " } \\
\text { après " lit fluidisé }\end{array}$ & $\begin{array}{l}0,160 \\
0,150\end{array}$ & $\begin{array}{l}0,215 \\
0,215\end{array}$ \\
\hline $\begin{array}{l}\text { Poudre séchée en } \\
\text { simple effet }\end{array}$ & en aval de la tour & 0,179 & 0,230 \\
\hline
\end{tabular}

sique, le séchage final en "lit fluidisé » et le séchage à double effet n'ont influé aucunement sur ces importantes caractéristiques des poudres de lait écrémé.

\section{IV.3. Groupes SH libres, teneur totale en groupes SH (thiol) et groupes SS (disulfure)}

Les groupes thiol et disulfure jouent un rôle important dans les conversions chimiques qui peuvent se produire entre certaines protéines. Il se peut que des modifications de la structure protéique ne soient pas perceptibles d'après les caractéristiques déjà décrites, mais se manifestent par la présence des groupes thiol et disulfure. C'est pourquoi l'attention s'est portée aussi, au cours de ces recherches, sur les groupes $\mathrm{SH}$ libres, la teneur totale en groupes $\mathrm{SH}$ et les groupes SS.

Les tableaux 5 et 6 présentent ces caractéristiques en relation avec les essais décrits sous IV,1 et IV,2. Il ressort clairement des deux tableaux que la présence des groupes thiol et disulfure n'est guère influencée par le séchage final en " lit fluidisé ». Il n'y a pas non plus 


\section{TABLEAU 5}

Influence du séchage à double effet et du séchage en " lit fluidisé 》 sur les nombres de $\mathrm{SH}$ libres et les teneurs en groupes thiol $(\mathrm{SH})$ et disulfure (SS).

Préchauffage de la concentration : $90^{\circ} \mathrm{C} / 30 \mathrm{~s}$

\begin{tabular}{|c|c|c|c|c|}
\hline $\begin{array}{l}\text { Température } \\
\text { de l'air sécheur en } \\
\text { " lit fluidisé " }\end{array}$ & Echantillonnage & $\begin{array}{c}\text { SH libre } \\
\mathrm{mM}\end{array}$ & $\begin{array}{l}\mathrm{SH} \text { total } \\
\mathrm{mg} / \mathrm{g} \\
\text { protéines }\end{array}$ & $\begin{array}{c}\mathrm{SS} \\
\mathrm{mg} / \mathrm{g} \\
\text { protéines }\end{array}$ \\
\hline 74 à $77^{\circ} \mathrm{C} / 4 \mathrm{~min}$ & $\begin{array}{l}\text { avant " lit fluidisé " } \\
\text { après " lit fluidisé " }\end{array}$ & $\begin{array}{l}10 \\
11,5\end{array}$ & $\begin{array}{l}0,185 \\
0,183\end{array}$ & $\begin{array}{l}1,87 \\
1,85\end{array}$ \\
\hline 81 à $83^{\circ} \mathrm{C} / 4 \mathrm{~min}$ & $\begin{array}{l}\text { avant " lit fluidisé " } \\
\text { après " lit fluidisé " }\end{array}$ & $\begin{array}{l}11,5 \\
16\end{array}$ & $\begin{array}{l}0,202 \\
0,182\end{array}$ & $\begin{array}{l}1,86 \\
1,87\end{array}$ \\
\hline 91 à $95^{\circ} \mathrm{C} / 4 \mathrm{~min}$ & $\begin{array}{l}\text { avant " lit fluidisé " } \\
\text { après " lit fluidisé " }\end{array}$ & $\begin{array}{l}12,5 \\
13\end{array}$ & $\begin{array}{l}0,195 \\
0,198\end{array}$ & $\begin{array}{l}1,82 \\
1,84\end{array}$ \\
\hline $\begin{array}{l}\text { Poudre séchée en } \\
\text { simple effet }\end{array}$ & en aval de la tour & 14 & 0,205 & 1,89 \\
\hline
\end{tabular}

\section{TABLEAU 6}

Influence du séchage à double effet du séchage en " lit fluidisé " sur les nombres de $\mathrm{SH}$ libres et les teneurs en groupes thiol (SH) et disulfure (SS).

Préchauffage de la concentration : $75^{\circ} \mathrm{C} / 30 \mathrm{~s}$

\begin{tabular}{|c|c|c|c|c|}
\hline $\begin{array}{l}\text { Température } \\
\text { de l'air sécheur en } \\
\text { " lit fluidisé » }\end{array}$ & Echantillonnage & $\underset{\mathrm{mM}}{\mathrm{SH} \text { libre }}$ & $\begin{array}{l}\text { SH total } \\
\text { mg/g } \\
\text { protéines }\end{array}$ & $\begin{array}{c}\mathrm{SS} \\
\mathrm{mg} / \mathrm{g} \\
\text { protéines }\end{array}$ \\
\hline 74 à $78^{\circ} \mathrm{C} / 4 \mathrm{~min}$ & $\begin{array}{l}\text { avant " lit fluidisé » } \\
\text { après « lit fluidisé » }\end{array}$ & $\begin{array}{l}1,5 \\
2\end{array}$ & $\begin{array}{l}0,169 \\
0,170\end{array}$ & $\begin{array}{l}2,18 \\
2,17\end{array}$ \\
\hline 85 à $90^{\circ} \mathrm{C} / 8 \mathrm{~min}$ & $\begin{array}{l}\text { avant " lit fluidisé » } \\
\text { après " lit fluidisé " }\end{array}$ & $\begin{array}{l}2,5 \\
4,5\end{array}$ & $\begin{array}{l}0,158 \\
0,152\end{array}$ & $\begin{array}{l}2,14 \\
2,16\end{array}$ \\
\hline 96 à $98^{\circ} \mathrm{C} / 4 \mathrm{~min}$ & $\begin{array}{l}\text { avant " lit fluidisé » } \\
\text { après « lit fluidisé " }\end{array}$ & $\begin{array}{l}1,5 \\
0,5\end{array}$ & $\begin{array}{l}0,160 \\
0,164\end{array}$ & $\begin{array}{l}2,18 \\
2,14\end{array}$ \\
\hline $\begin{array}{l}\text { Poudre séchée en } \\
\text { simple effet }\end{array}$ & en aval de la tour & 1 & 0,157 & 2,14 \\
\hline
\end{tabular}


de différence entre la poudre obtenue par séchage à simple effet et celles qui ont subi le séchage à double effet.

\section{IV.4. Stabilité à la chaleur du lait reconstitué et du lait recombiné}

Il ressort de l'ensemble des données ci-dessus que, dans les conditions d'essai décrites, le séchage en " lit fluidisé » n'a eu pratiquement aucune influence sur les caractéristiques étudiées. On peut donc escompter que les caractéristiques fonctionnelles changeront peu, pour autant qu'elles soient déterminées par des propriétés chimiques. Les stabilités à la chaleur du lait reconstitué (9 p. 100 d'extrait sec) et du lait recombiné (18 p. 100 d'extrait sec) ont été déterminées à respectivement 135 et $120^{\circ} \mathrm{C}$, à titre d'application dans ce contexte. Les tableaux 7 et 8 reproduisent les résultats moyens relatifs aux séries d'essai déjà mentionnées. Ils montrent que le "lit fluidisé » et le séchage à double effet n'ont pas d'influence, ni pour le lait reconstitué ni pour le lait recombiné. On peut donc admettre d'une façon générale que les échauffements appliqués en " lit fluidisé ", tels qu'ils se déroulent dans le séchage à double effet, n'ont pas d'influence indésirable sur les caractéristiques, décrites ici, des poudres de lait écrémé.

\section{TABLEAU 7}

Influence du séchage à double effet et du séchage en " lit fluidisé » sur la stabilité à la chaleur du lait reconstitué et du lait recombiné.

Préchauffage de la concentration : $90^{\circ} \mathrm{C} / 30 \mathrm{~s}$

\begin{tabular}{|c|c|c|c|}
\hline \multirow{2}{*}{$\begin{array}{l}\text { Température de l'air } \\
\text { sécheur en } \\
\text { « lit fluidisé " }\end{array}$} & \multirow{2}{*}{ Echantillonnage } & \multicolumn{2}{|c|}{ Stabilité à la chaleur } \\
\hline & & $\begin{array}{l}9 \text { p. } 100 \\
135^{\circ} \mathrm{C}\end{array}$ & $\begin{array}{l}18 \text { p. } 100 \\
120^{\circ} \mathrm{C}\end{array}$ \\
\hline 74 à $77^{\circ} \mathrm{C} / 4 \mathrm{~min}$ & $\begin{array}{l}\text { avant " lit fluidisé " } \\
\text { après " lit fluidisé " }\end{array}$ & $\begin{array}{l}16 \min \\
15 \min 30 \mathrm{~s}\end{array}$ & $\begin{array}{l}17 \min 30 \mathrm{~s} \\
18 \min 30 \mathrm{~s}\end{array}$ \\
\hline 81 à $83^{\circ} \mathrm{C} / 4 \mathrm{~min}$ & $\begin{array}{l}\text { avant " lit fluidisé " } \\
\text { après " lit fluidisé " }\end{array}$ & $\begin{array}{l}16 \min 30 \mathrm{~s} \\
16 \min \end{array}$ & $\begin{array}{l}20 \mathrm{~min} \\
20 \mathrm{~min}\end{array}$ \\
\hline 91 à $95^{\circ} \mathrm{C} / 4 \min$ & $\begin{array}{l}\text { avant " lit fluidisé " } \\
\text { après " lit fluidisé " }\end{array}$ & $\begin{array}{l}15 \min 30 \mathrm{~s} \\
16 \min 30 \mathrm{~s}\end{array}$ & $\begin{array}{l}19 \min \\
20 \mathrm{~min}\end{array}$ \\
\hline $\begin{array}{c}\text { Poudre séchee en simple } \\
\text { effet }\end{array}$ & en aval de la tour & $16 \mathrm{~min}$ & $20 \mathrm{~min}$ \\
\hline
\end{tabular}




\section{TABLEAU 8}

Influence du séchage à double effet et du séchage en " lit fluidisé " sur la stabilité à la chaleur du lait reconstitué et du lait recombiné.

Préchauffage de la concentration : $75^{\circ} \mathrm{C} / 30 \mathrm{~s}$

\begin{tabular}{|c|c|c|c|}
\hline \multirow{2}{*}{$\begin{array}{c}\text { Température de l'air } \\
\text { sécheur en } \\
\text { " lit fluidisé " }\end{array}$} & \multirow{2}{*}{ Echantillonnage } & \multicolumn{2}{|c|}{ Stabilité à la chaleur } \\
\hline & & $\begin{array}{l}9 \text { p. } 100 \\
135^{\circ} \mathrm{C}\end{array}$ & $\begin{array}{l}18 \text { p. } 100 \\
120^{\circ} \mathrm{C}\end{array}$ \\
\hline 74 à $78^{\circ} \mathrm{C} / 4 \mathrm{~min}$ & $\begin{array}{l}\text { avant " lit fluidisé " } \\
\text { après " lit fluidisé " }\end{array}$ & $\begin{array}{l}19 \min 30 \mathrm{~s} \\
21 \min 30 \mathrm{~s}\end{array}$ & $\begin{array}{l}22 \min 30 \mathrm{~s} \\
23 \min 30 \mathrm{~s}\end{array}$ \\
\hline 85 à $90^{\circ} \mathrm{C} / 8 \mathrm{~min}$ & $\begin{array}{l}\text { avant " lit fluidisé " } \\
\text { après " lit fluidisé " }\end{array}$ & $\begin{array}{l}19 \min 30 \mathrm{~s} \\
19 \min 30 \mathrm{~s}\end{array}$ & $25 \min 30 \mathrm{~s}$ \\
\hline 96 à $98^{\circ} \mathrm{C} / 4 \mathrm{~min}$ & $\begin{array}{l}\text { avant « lit fluidisé " } \\
\text { après " lit fluidisé » }\end{array}$ & $\begin{array}{l}20 \min 30 \mathrm{~s} \\
20 \mathrm{~min} 30 \mathrm{~s}\end{array}$ & $\begin{array}{l}22 \min \\
24 \min \end{array}$ \\
\hline $\begin{array}{c}\text { Poudre séchée en simple } \\
\text { effet }\end{array}$ & en aval de la tour & $21 \mathrm{~min}$ & $26 \mathrm{~min}$ \\
\hline
\end{tabular}

\section{RESUME ET CONCLUSION}

Cet article donne un aperçu des résultats relatifs à quelques caractéristiques chimiques importantes, obtenus au cours du séchage à double effet de poudres de lait écrémé. On peut conclure de ce travail que, comparativement au processus de séchage classique, le séchage à double effet, considéré dans son ensemble, et le séchage en « lit fluidisé » considéré seul n'ont eu, dans les conditions décrites, aucune influence sur les caractéristiques chimiques étudiées. Les principales caractéristiques retenues dans cette étude sont celles qui sont à la base de la classification des poudres en fonction des traitements thermiques appliqués : la teneur en protéines sériques non dénaturées (WPNI, indice de caséine, électrophorèse), la teneur en chlorhydrate de cystéine (propriétés réductrices) et l'intensité de la réaction de Maillard, le nombre de groupes $\mathrm{SH}$ libres et les teneurs totales en groupes SH et en groupes SS (cystéine et cystine). La stabilité à la chaleur après reconstitution confirme l'observation générale, que le séchage à double effet n'exerce aucune influence positive ou négative sur les caractéristiques étudiées.

Les résultats ont été obtenus dans des essais à l'échelle pilote. On retrouverait probablement les mêmes tendances dans des condi- 
tions de fabrication industrielles, les valeurs absolues mentionnées ici pouvant cependant différer d'une installation à l'autre.

\section{Remerciements}

L'auteur remercie M. H. De Ridder, ingénieur industriel, membre du Groupe de Travail pour l'amélioration de la qualité des produits laitiers, de sa précieuse collaboration.

\section{S u $\mathrm{m}$ m a ry}

THE CHEMICAL PROPERTIES OF TWO-STAGE DRIED SKIM MILK POWDER

A survey is given of the results obtained for some important chemical characteristics of skim milk powder after two-stage drying.

It can generally be concluded that both two-stage drying and fluid bed drying do not affect, under the given conditions and compared to the classic drying procedure, the investigated chemical characteristics of the skim milk powders. The main characteristics studied were: the heat classification by following the content of non-denaturated whey proteins (whey protein nitrogen index, caseine number and electrophoresis), the cysteine hydrochloride content (reducing characteristics) and the Maillard reaction, the free $\mathrm{SH}$ groups and the total content of SH and SS groups (cysteine and cystine). The heat stability after reconstitution confirms the general finding that two-stage drying does not affect, neither positively nor negatively, the characteristics investigated.

The results were obtained during experiments on a pilot scale. Under industrial circumstances the same tendencies are likely to be found. The absolute values mentioned in this study may, however, vary from plant to plant.

Reçu pour publication en septembre 1980.

\section{Bibliographie}

[1] ADMI (1971). - Determination of undenatured whey protein nitrogen in non-fat dry milk. Standards for Grades of Dry Milks. American Dry milk Institute, Bulletin, 916, 2, 49.

[2] Assenat (L.) (1967). - Contribution à l'étude d'une méthode d'identification des laits et fromages au moyen de l'électrophorèse sur gel de polyacrylamide. Le Lait, 47, 393.

[3] Crowe (L. K.), Jenness (R.) and Coulter (S. T.) (1948). - The reducing capacity of milk and milk products as measured by a modified ferricyanide method. J. Dairy Sci., 31, 595. 
[4] Davies (D. T.) and White (J. C. D.) (1966). - The stability of milk protein to heat. I. Subjective measurement to heat stability of milk. J. Dairy Res., 33, 67.

[5] De Vilder (J.) (1980). - Propriétés physiques de la poudre de lait entier fabriquée selon la méthode de séchage à double effet. Le Lait, 599-600, 561-573.

[6] De Vilder (J.) et Martens (R.) (1976). - La classification des poudres de lait écrémé en fonction des traitements thermiques appliqués. Revue de l'Agriculture, 4, 877.

[7] Eggum (B. O.), Nielsen (H. E.) und Rasmussen (F. L.) (1970). - Der Einfluss der Lagerung auf die Proteinqualität von Magermilchpulver. Z. Tierphysiol., Tierernähr. Futtermittelk, 27, 1, 18.

[8] Henry (K. M.), KoN (S. K.), LeA (C. H.) and White (J. C. D.) (1948), Deterioration on storage of dried skim milk. J. Dairy Res., 15, 292.

[9] KEENEY (M.) (1963). - Chemical Characterization of off-flavors in concentrated and nonfat dried milk. J. Dairy Sci., 46, 254.

[10] KeEney (M.) and Bassette (R.) (1959). - Detection of intermediate compounds in the early stages of browning reaction in milk products. J. Dairy Sci., 42, 945.

[11] Kumetat (K.), Beeby (R.) and Morris (A. K.) (1956). - A note on the reducing groups of «Instant» milk powders. Australian J. Dairy Technol., $11,113$.

[12] Lyster (R. L. J.) (1964). - The free and masked sulphydryl groups of heated milk and milk powder and a new method for their determination. J. Dairy Res., 31, 41.

[13] ManN (J. B.) and Mc Intire (J. M.) (1961). - Comparison of Flavor and Some Chemical Properties of Instantized and Noninstantized Nonfat Dry milk. J. Dairy Sci., 44, 851.

[14] Mrowetz (G.), Klostermeyer (H.) und Thomasow (J.) (1972). - Thiol- und Disulfidgruppen in Milchprodukten. Bestimmung und Ergebnisse. $Z$. Lebensmittelunters. u. Forschung, 149, 341.

[15] RobBertSen (T.) and RADEMA (L.) (1975). - An improved method for the determination of the reducing capacity of milk powder. Neth. Milk Dairy J., 29, 354.

[16] Samuelsson (E. G.) and Nielsen (P.) (1970). - Formation of hydroxymethylfurfural caused by heat-treatment of milk. Milchwissenschaft, 25, 541 . 